\title{
Recentes Avanços na Pesquisa Experimental em Cardiologia
}

\section{Overview of Recent Advances in Experimental Cardiovascular Research}

\author{
Luana U. Pagan, ${ }^{10}$ Mariana J. Gomes, ${ }^{2}$ Marina P. Okoshi' ${ }^{10}$ \\ Faculdade de Medicina de Botucatu - Universidade Estadual Paulista (UNESP), ${ }^{1}$ Botucatu, SP - Brasil \\ Brigham and Women's Hospital - Harvard Medical School, ${ }^{2}$ Boston, MA - EUA
}

A pesquisa básica é de grande importância para fomentar e ampliar o conhecimento nas mais diversas áreas. A prática da cardiologia clínica modificou-se substancialmente em resposta aos avanços das pesquisas experimentais das últimas décadas, as quais possibilitaram maior entendimento dos mecanismos moleculares envolvidos nas doenças cardiovasculares. Consequentemente, novas ferramentas foram introduzidas para diagnóstico e novos fármacos tiveram suas indicações definidas para o tratamento das doenças cardiovasculares. ${ }^{1}$

A observação dos artigos publicados nos Arquivos Brasileiros de Cardiologia nos últimos anos mostra grande avanço da produção científica na área básica, tanto de artigos originários de investigadores brasileiros como de pesquisadores de várias nações. Adicionalmente, verifica-se a diversidade de origem da produção científica nacional relacionada a campos do conhecimento; na última década, o jornal teve expressivo aumento da quantidade de artigos de áreas como educação física, fisioterapia, nutrição, biologia e biomedicina, dentre outras. Neste editorial, comentaremos sobre estudos recentemente publicados nos Arquivos Brasileiros de Cardiologia na área de pesquisa básica.

A prática de exercícios tem sido considerada cada vez mais importante na prevenção e no tratamento de doenças cardiovasculares, particularmente quando se leva em conta o envelhecimento da população. ${ }^{2}$ Consequentemente, o papel do exercício físico na evolução das doenças cardiovasculares teve destaque na produção científica nacional e internacional nos últimos anos. Artigos recentemente publicados nos $\mathrm{ABC}$ mostraram que o exercício físico contribui para o equilíbrio redox e inflamatório no coração em situações de agressão sistêmica, como a obesidade ${ }^{3}$ e a ovariectomia associada a nocaute do receptor da lipoproteína de baixa densidade. ${ }^{4}$ Além disso, foi relatado que, na cardiopatia diabética, o exercício estimula a angiogênese miocárdica. ${ }^{5}$ Mesmo atividades consideradas passivas, como a vibração de corpo inteiro, tiveram efeitos benéficos em ratos, como o aumento da tolerância à isquemia miocárdica. ${ }^{6} \mathrm{O}$ conjunto dos

\section{Palavras-chave}

Doenças Cardiovasculares/tendências; Exercício; Insuficiência Cardíaca; Mecanismos Moleculares/tendências; Domínios Científicos; Atividades Científicas e Tecnológicas/tendências.

Correspondência: Marina P. Okoshi •

Faculdade de Medicina de Botucatu - Rubião Junior, S/N. CEP 18618-687, Botucatu, SP - Brasil

E-mail: mpoliti@fmb.unesp.br

DOI: https://doi.org/10.36660/abc.20200835 resultados contribui para o entendimento dos mecanismos pelos quais o exercício tem efeitos benéficos na prevenção e no tratamento das doenças cardiovasculares.

Terapia nutricional é outra estratégia extensamente abordada na cardiologia. ${ }^{7}$ Um estudo recentemente publicado destacou os efeitos antioxidantes do açaí e a melhora do metabolismo energético no modelo global de isquemiareperfusão em ratos, que foram independentes de alterações na função ventricular esquerda. ${ }^{8}$ Outro componente avaliado experimentalmente foi a gordura. Muniz et al. ${ }^{9}$ observaram que dieta rica em banha aumenta o peso corporal sem, no entanto, levar a dislipidemia em ratos. Por outro lado, alimentação rica em banha e em colesterol induz dislipidemia e danos graves ao fígado. ${ }^{9}$

Além das potenciais estratégias não farmacológicas descritas, novas indicações de medicamentos foram contempladas nos estudos publicados nos ABC. Ramezani-Aliakbari et al. ${ }^{10}$ avaliaram o uso da trimetazidina em ratos com cardiomiopatia diabética. O fármaco, comumente utilizado para melhorar o metabolismo miocárdico na insuficiência coronariana, reduziu o grau de hipertrofia miocárdica e melhorou parâmetros eletrocardiográficos e de função ventricular. Outro medicamento avaliado foi o losartan, um bloqueador do receptor tipo 1 da angiotensina II, que melhorou a função miocárdica de ratos com obesidade induzida por dieta. ${ }^{11}$

No campo da revascularização miocárdica, foi observado que a administração de rapamicina em combinação com $\alpha$-cianoacrilato foi superior para manter a patência vascular ao uso isolado de cada um dos fármacos em enxertos vasculares de ratos. ${ }^{12}$ Os efeitos positivos pareceram ligados a diminuição do espessamento intimal, da proliferação celular e da resposta inflamatória no enxerto. ${ }^{12}$

Finalmente, foram publicados estudos relacionados à investigação de fatores agravantes de doenças cardiovasculares. Vassallo et al. ${ }^{13}$ observaram que a exposição ao mercúrio agrava a hipertensão arterial sistêmica e aumenta a atividade plasmática da enzima conversora da angiotensina e o estresse oxidativo miocárdico de ratos espontaneamente hipertensos. Outro fator de risco para doenças cardiovasculares abordado foi o estresse físico, ${ }^{14,15}$ cuja indução durante o período prénatal resultou em alterações sexo-específicas na expressão gênica de receptores adrenérgicos $\beta 1$ da prole adulta de ratos. ${ }^{14}$ Por outro lado, a aplicação de estresse físico 60 minutos antes da indução de isquemia-reperfusão teve efeitos benéficos, como a redução da área do infarto e a melhora da função ventricular em ratos. ${ }^{15}$

Apesar do aumento nas informações sobre vias de sinalização moduladas pelo exercício físico, dos efeitos sistêmicos e cardiovasculares de diferentes terapias nutricionais 
e dos novos usos de fármacos para prevenção e tratamento de doenças cardiovasculares, ainda há um longo caminho até que o conhecimento possa ser incorporado à prática clínica. Espera-se que o avanço na medicina translacional possa contribuir para a redução do tempo entre o conhecimento básico e sua aplicação clínica. Para isso, os Arquivos Brasileiros de Cardiologia têm importante papel na publicação de artigos com relevância e importância científica em todas as áreas do conhecimento relacionadas à cardiologia. Adicionalmente, o

\section{Referências}

1. Virani SS, Alonso A, Benjamin EJ Bittencourt MS, Callaway CW, Carson AP, et al. American Heart Association Council on Epidemiology and Prevention Statistics Committee and Stroke Statistics Subcommittee. Heart disease and stroke statistics-2020 update: a report from the American Heart Association. Circulation. 2020; 141(9):e139-e596.

2. Gomes MJ, Martinez PF, Pagan LU, Damatto RL, Cezar MD, Lima AR, et al. Skeletal muscle aging: influence of oxidative stress and physical exercise. Oncotarget 2017; 8(12): 20428-20440.

3. Effting PS, Brescianini SMS, Sorato HR, Fernandes BB, Fidelis GDSP, Silva PR, et al. Resistance exercise modulates oxidative stress parameters and TNF- $\alpha$ content in the heart of mice with diet-induced obesity. Arq Bras Cardiol 2019; 112(5): 545-552.

4. Brianezi L, Ornelas E, Gehrke FS, Fonseca FLA, Alves BCA, Sousa LVA, et al. Effects of physical training on the myocardium of oxariectomized LDLR knockout mice: MMP 2/9, collagen I/III, inflammation and oxidative stress. Arq Bras Cardiol 2020; 114(1): 100-105.

5. Naderi R, Mohaddes G, Mohammadi M, Alihemmati A, Khamaneh A, Ghyasi $\mathrm{R}$, et al. The effect of garlic and voluntary exercise on cardiac angiogenesis in diabetes: the role of MiR-126 and MiR-210. Arq Bras Cardiol. 2019; 112(2):154-62.

6. Shekarforoush S, Naghii MR. Whole-body vibration training increases myocardial salvage against acute ischemia in adult male rats. Arq Bras Cardiol $2019 ; 112(1): 32-37$.

7. Carson JAS, Lichtenstein AH, Anderson CAM, Appel LJ, Kris-Etherton PM, Meyer KA, et al. American Heart Association Nutrition Committee of the Council on Lifestyle and Cardiometabolic Health; Council on Arteriosclerosis, Thrombosis and Vascular Biology; Council on Cardiovascular and Stroke Nursing; Council on Clinical Cardiology; Council on Peripheral Vascular Disease; and Stroke Council. Dietary cholesterol and cardiovascular risk: a science advisory from the American Heart Association. Circulation. 2020; 141(3):e39-e53. periódico tem promovido a interprofissionalização e o debate científico de qualidade, integrando diferentes profissionais envolvidos na prevenção e nos cuidados de doenças do sistema cardiovascular.

\section{Agradecimentos}

FAPESP (Proc. n. 2018/07048-2) e CNPq (Proc. n. 310876/2018-4).

8. Alegre P, Mathias L, Lourenço MA, Santos PP, Gonçalves A, Fernandes AA, et al. Euterpe oleracea mart. (açaí) reduces oxidative stress and improves energetic metabolism in myocardial ischemia-reperfusion injury in rats. Arq Bras Cardiol. 2020; 114(1):78-86.

9. Muniz LB, Alves-Santos AM, Camargo F Martins DB, Celes MRN, Naves MMV. High-lard and high-cholesterol diet, but not high-lard diet, leads to metabolic disorders in a modified dyslipidemia model. Arq Bras Cardiol. 2019; 113(5):896-902.

10. Ramezani-Aliakbari F, Badavi M, Dianat M Mard SA, Ahangarpour A. The effects of trimetazidine on QT-interval prolongation and cardiac hypertrophy in diabetic rats. Arq Bras Cardiol 2019; 112(2): 173-178.

11. Oliveira Jr AS, Muzili NA, Carvalho MR, Ota GE, Morais CS, Vieira LFC, Ortiz MO, Campos DHS, Cezar MDM, Okoshi MP, Okoshi K, Cicogna AC, Martinez PF. AT1 receptor blockade improves myocardial functional performance in obesity. Arq Bras Cardiol 2020; 115: 17-28.

12. Tianshu-Chu, Congrong-Gao, Zhiwei-Zhao, Fei-Ling, Ayu-Sun, YuanbiaoZheng, et al. Rapamycin combined with $\alpha$-cyanoacrylate contributes to inhibiting intimal hyperplasia in rat models. Arq Bras Cardiol 2019; 112(1): 3-10.

13. Vassallo DV, Simões MR, Giuberti K, Azevedo BF, Ribeiro Junior RF, Salaices $M$, et al. Effects of chronic exposure to mercury on angiotensin-converting enzyme activity and oxidative stress in normotensive and hypertensive rats. Arq Bras Cardiol. 2019; 112(4):374-80.

14. Jevjdovic T, Dakic T, Kopanja S, Lakic I, Vujovic P, Jasnic N, et al. Sex-related effects of prenatal stress on region-specific expression of monoamine oxidase $A$ and $\beta$ adrenergic receptors in rat hearts. Arq Bras Cardiol. 2019; 112(1):67-75.

15. Imani A, Parsa H, Chookalaei LG, Rakhshan K, Golnazari M, Faghihi M. Acute physical stress preconditions the heart against ischemia/reperfusion injury through activation of sympathetic nervous system. Arq Bras Cardiol. 2019; 113(3):401-8. 